IP Periodica Polytechnica Civil Engineering

\author{
59(1), pp. 9 2014 \\ DOI: $10.3311 /$ PPci.7341 \\ Creative Commons Attribution (1) \\ RESEARCH ARTICLE
}

\section{Multi Objective Particle Swarm Optimization (MOPSO) for Size and Shape Optimization of 2D Truss Structures}

Seyed Saeed Hosseini, Sajad Ahmad Hamidi, Motahar Mansuri, Ali Ghoddosian Received 09-02-2014, revised 22-10-2014, accepted 26-11-2014

\begin{abstract}
This paper covers optimization techniques for trusses to find the most efficient cross sections and configuration of joints. The improvement will be achieved by applying changes in one or both of these parameters. Objective functions for optimization are weight and the deflection of the truss's joints. For optimization of both weight and deflection the MOPSO method is used. This is a powerful method that enables the optimization of huge trusses. The former methods for optimization of the shape or size of the trusses, was done separately and as a single objective while this paper covers a new way via multi objective methods. For proofing the ability of the represented method in this paper, some standard examples are compared. The comparison of the results shows good accuracy and desirable verity of pareto front.
\end{abstract}

\section{Keywords}

Truss · Multi objective optimization · Size optimization · MOPSO $\cdot$ Shape optimization

\section{Seyed Saeed Hosseini}

Department of Mechanical Engineering, Semnan Branch, Islamic Azad University, Semnan, Iran

e-mail: hosseiny.saeed@yahoo.com

Sajad Ahmad Hamidi

Civil and Environmental Engineering, University of Wisconsin-Milwaukee, 3200 North Cramer St., Milwaukee, USA

e-mail: hamidi@uwm.edu

\section{Motahar Mansuri}

Department of Mechanical Engineering, Semnan Branch, Islamic Azad University, Semnan, Iran

e-mail: motaharmansouri@gmail.com

\section{Ali Ghoddosian}

Faculty of Mechanical Engineering, Semnan University, P.O. Box: 3513119111, Semnan, Iran

e-mail: aghoddosian@semnan.ac.ir

\section{Introduction}

Designers have been thinking about decreasing the weight of the structure without having any unfavorable effect on its behavior for many years. A good engineering design aims to reduce the cost of design and construction by reduction the martial used in a project as well as reduction in calculation time. However, presenting a fundamental theory in this field goes back to the researches done by Michel at the early 20th century. By considering the obvious and fixed state of the load trait points and backrests/bases in 2 - dimensional space, he established an analytical method for optimization plan for trusses which have only one form of loading and the tension limitation is considered in them. Michel structure was statically specified and stable only for the intended loading form. This structure due to having an infinite number of members is rarely applicable in engineering plans and can be used only as a guide in truss' body optimization plan [1, 2]. Nowadays two main optimization methods are used for solving the problems. In the first optimization method the gradient information is used for searching around the primary starting point. The second one consists of meta-heuristic techniques. Meta-heuristic optimization algorithms are powerful tools for solving engineering optimization problems. These algorithms have been used by different people and in different subjects. For example, they have been used in mechanical engineering and electronic engineering by Kim Park [3] and Woo et al [4] respectively. Some of the Meta-heuristic algorithms are as follow:

1 Genetic algorithm [5]

2 Charged system search [6]

3 Particle swarm optimization [7]

4 ICACO algorithm [8]

One of the problems that Meta-heuristic algorithms are applied in is truss optimization problem. Trusses are among the important structures and are usually used as ceiling, power transfer towers, bridges, and so on. Truss optimization problems are categorized in three main groups: The first group is size optimization in which the cross section is considered as the designing variable. The second group is form optimization in which 
the configuration of the truss' nodes is considered as the designing variable. The third group is topology optimization in which the truss' link is optimized. It is worth mentioning that in each optimization problem there are constraints, like stress, cross sections or other parameters that should be considered. The general form of optimization problem is as follows:

$$
\begin{aligned}
& \text { Minimize } f(x)=\sum_{i=1}^{n} A_{i} L_{i}(X) \\
& \text { Subject to: } A_{\text {min }} \geq A_{i} \geq A_{\text {max }} \\
& \sigma_{\text {min }} \geq \sigma_{i} \geq \sigma_{\text {max }} \\
& \delta_{\text {min }} \geq \delta_{i} \geq \delta_{\text {max }}
\end{aligned}
$$

In this study the Multi objective particle swarm optimization (MOPSO) is applied for shape and size optimization of truss structures and the ability and accuracy of method is compared with other popular optimization methods.

\section{Particle Swarm Optimization Algorithm}

Particle Swarm Optimization (PSO) algorithm which is modeled based on birds' collective movement, optimizes the objective function through cooperation of a set of particles in the search space. This algorithm was presented by Kennedy and Eberhart in 1995 [9]. It is considered as one of the one- objective optimization methods which optimizes the problems through zero order method (no derivatives). PSO algorithm can be explained as follows:

1 Setting the primary population (all particles)

2 Determining the best particle and the best memory of each particle

3 Updating the speed and state of all the particles

4 Determining the best particle and the best memory of each particle

5 In case of not meeting the finishing condition go to 3 , otherwise (the end)

In the PSO method, each particle obeys the following relations to achieve the new state:

$$
x(t+1)=x(t)+v(t+1)
$$

$$
v(t+1)=w v(t)+c_{1} r_{1}(p(t)-x(t))+c_{2} r_{2}(g(t)-x(t))
$$

In which:

$p(t) \quad$ is the best state of each particle

$g(t) \quad$ is the best state they have experienced

$c_{1}, c_{2}$ are impression coefficients

$r_{1}, r_{2}$ are random numbers between 0 and 1

$t$ is algorithm iteration

$w \quad$ Coefficient to control the effects of $\mathrm{v}(\mathrm{t})$

$c_{1}$ and $c_{2}$ are coefficients for vectors $p(t)-x(t)$ and $g(t)-x(t)$ that show the position of particles and the vectors lengths.

\section{Multi Objective Optimization}

In optimization process, there are problems with several objective functions. In this type of problems several parameters should be optimized simultaneously. These are called multi objective optimization problems. In these types of optimization problems, objective functions are in contradiction with each other. For example in a truss structure, by decreasing the cross section of the elements the weight is decreased while the tension of members and consequently the deflections are increased. Following a brief explanation about the concepts related to Multi Objective Optimization methods are discussed.

\subsection{Domination}

In multi objective optimization the concept of domination is used for comparing the points. If all the $X_{1}$ solution are not worse than all solution $X_{2}$ in all objectives, or if all the $X_{1}$ solutions are equal to $X_{2}$ but only in one case or one dimension $X_{1}$ is better than $X_{2}$, then it can be said that $X_{1}$ will dominate $X_{2}$. The mathematical expression of the domination concept is as follows:

$$
\begin{aligned}
& x_{1} \text { dominate } x_{2} \text { if: } \\
& f\left(x_{1}\right) \geq f\left(x_{2}\right) \\
& f\left(x_{1}\right)<f\left(x_{2}\right)
\end{aligned}
$$

\subsection{Pareto front}

In solving the multi objective problems it is not possible to announce a certain point as the answer. Because if we find a point in which one of the target functions is minimum, there is another target function which is not minimum at this point. Therefore, the answer to a multi objective optimization problem is not a point but a curve in which every point is optimized according to just one of the objective functions. Also, these points will never dominate each other.

\subsection{Repository}

Repository members are non-dominated members of the problem which as a repository gathers the best points, achieved during the stages of performing optimization algorithm. To select these members two conditions are considered:

1 Not being dominated by the repository members

2 The most dispersion of the repository members

\section{Multi Objective Particle Swarm Optimization algo- rithm}

The Multi Objective Particle Swarm Optimization or MOPSO algorithm is taken from PESA2 algorithm and the operators of the PSO method are used instead of the genetic operators. This algorithm was presented by Coello in 2004 and in comparison with NSGA II [17]. Method has a less programming volume and 
therefore a higher speed by the software [10]. In the MOPSO algorithm, the objective space tabulation is used in order to have the most dispersion for the repository members. First, we tabulate the space in which the repository members are located, then to select the leader by the particles we think about a way in which the probability of choosing those cells which contain less population is more and vice versa. Therefore, the particles are inclined toward the less populated cells and it leads to the homogeneity of the population of all the cells which consequently results in the homogeneity of the repository members' dispersion. An example of the tabulating the target space along with the repository members and population members is presented in Fig. 1. In this figure the members' population and repository members are seen in stars and crosses, respectively. In this figure each repository member dominates all members but none of the repository members are dominated by another repository member. In order to delete the additional members of the repository, we select those members with the least distance from each other. By this, the repository members' dispersion factor is maintained. This paper introduces a proposal to extend the heuristic method called "particle swarm optimization" (PSO) to deal with multi objective optimization problems. Our approach uses the concept of Pareto dominance to determine the flight direction of a particle and it maintains previously found non-dominated vectors in a global repository that is later used by other particles to guide their own flight. The approach is validated using several standard test functions from the literature. Our results indicate that our approach is highly competitive with current evolutionary multi objective optimization techniques [16].

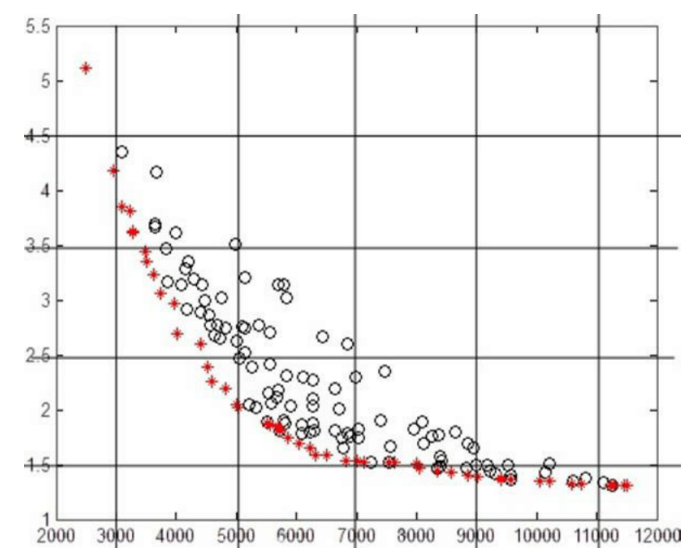

Fig. 1. Presentation of the tabulating the objective space, population members and repository members in MOPSO algorithm

For the purpose of trusses' multi objective optimization based on MOPSO method, each particle is considered as a complete truss in this algorithm and the state of this particle is the same as the members' cross section and the coordinate of the nodes. In order to calculate the amount of the function, we have used the limited elements relationships. By entering the members' cross section and the coordinate of the nodes it is possible to obtain the weight of the truss or the nodes' replacements or the average of the tension or any other features as the amount of the function.
To perform the MOPSO algorithm, the stress of any individual members of each truss is inspected in terms of stress and strain and if the stress is violated in one of the members, once again a new state is considered for the particles.

\section{Examples}

In this section, several examples are presented in order to introduce and express the efficiency of the MOPSO method. There are three main categories in truss optimization; namely size, shape and topology optimization. In the first category, the cross sectional areas are variable of design and minimum weight or cost is the target of optimization. In second category the nodal coordinates of a truss are the deign variables and the weight or cost is the goal of optimization. In both methods there are constraints like displacements of nodes or allowable stresses. In topology optimization the connectivity of structure is optimized. It should be noted that the topology of all the structures are the same and only the cross section areas and the position of the nodes are assumed as design variables in these problems. We use FEM to calculate the stress and deflection in the examples. For this purpose we developed a MATLAB code to analyze numerically the trusses.

\subsection{The multi objective optimization of the size of a 10-bar truss}

We consider a 10-bar truss as presented in Fig. 2 and mentioned in the reference [11]. The objective functions of the problems are minimizing the structure's weight and the vertical displacement of the $6^{\text {th }}$ node. Minimum and maximum cross section of the members is 0.64516 and $193.548 \mathrm{~cm}^{2}$, respectively. The external force $p=-444.8 \mathrm{KN}$ is put on the $5^{\text {th }}$ and $6^{\text {th }}$ nodes in $\mathrm{Y}$ direction. The maximum allowed stress of the members is $\pm 172.375 \mathrm{MPa}$. Modulus of elasticity is $E=68.95 \mathrm{GPa}$. The objective functions of the problem are minimizing the volume of the whole structure and also minimizing the vertical displacement of the $6^{\text {th }}$ node. The constraint of the problem is the maximum allowed stress of the members.

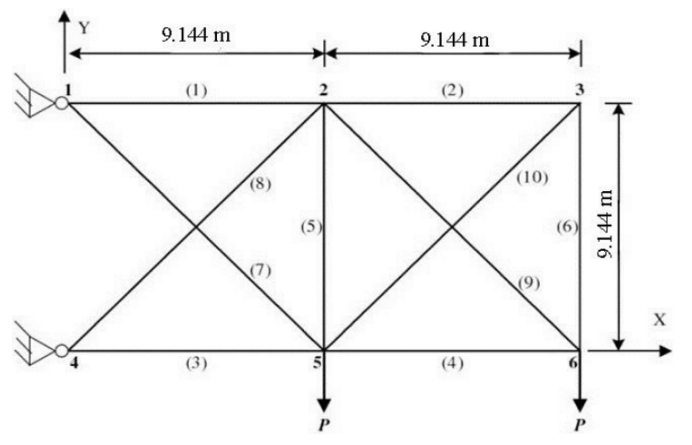

Fig. 2. 10-bar truss for the first problem

In Fig. 3, the results of this method are compared with those of the presented methods in the reference [11]. As it can be seen in Fig. 3, the results of MOPSO method completely conformed to other methods and its Pareto curve is placed on the Pareto curve 
of other methods. It should be mentioned that in this example of the truss' size optimization is presented by considering several objective functions.

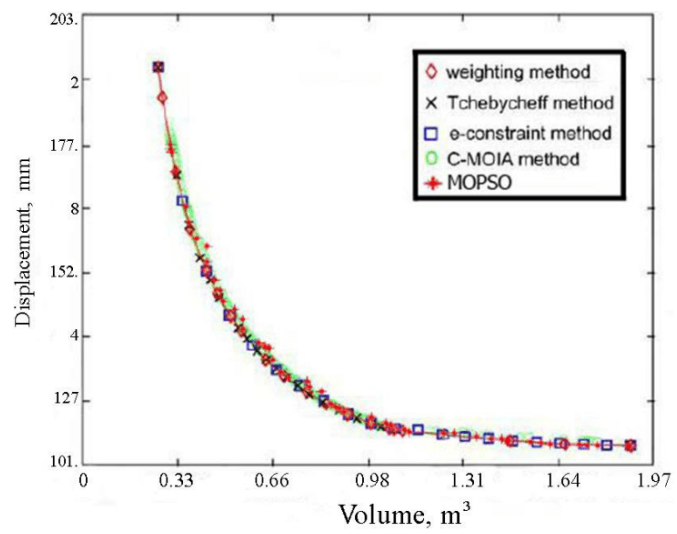

Fig. 3. Comparison of the results of MOPSO for the first problem with other methods

\subsection{Michel structure}

This structure which is often used for investigating the topology optimization methods has recently been used for form optimization through truss model for decreasing the structure's weight [12,13] and through skeleton model for decreasing the maximum bending moment and achieving the least maximum stress by evolutionary transfer method [14]. In this paper, it is used for form optimization of the structure. Michel structure which is shown in Fig. 4 bears the vertical load $p=200 \mathrm{KN}$ at its center. The elasticity module of all the members is $E=2.1 \times 10^{11} \mathrm{~Pa}$ and their density is $\rho=7800 \mathrm{~kg} / \mathrm{m}^{3}$. Also, the internal cross section of the members is considered as $10 \mathrm{~cm}^{2}$. In optimization the nodes coordinates are the design variable.

In order to optimize the form of the truss, third and $7^{\text {th }}$ nodes can move in the $\mathrm{X}$ direction but they won't have any displacement in $\mathrm{Y}$ direction. The $4^{\text {th }}, 5^{\text {th }}$ and $6^{\text {th }}$ nodes can also move in $\mathrm{Y}$ direction and have a fixed state in $\mathrm{X}$ direction. The first, second and $8^{\text {th }}$ nodes have no capability for state change. We want to optimize the weight and vertical deflection of node 1 through truss' shape optimization. One of the optimized trusses is shown in Fig. 5 .

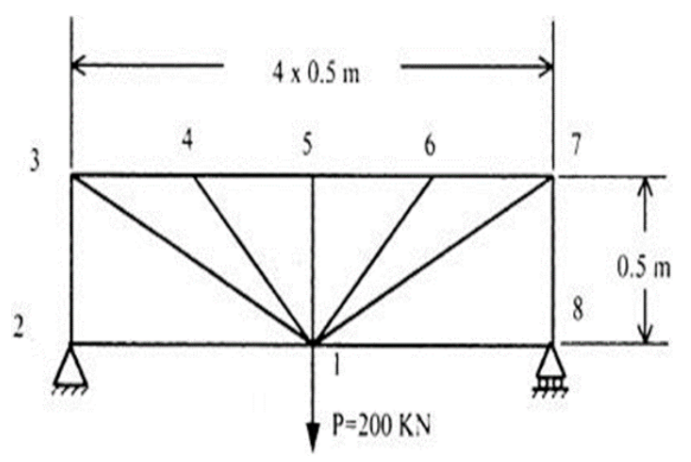

Fig. 4. Michel structure

As it can be seen in Fig. 5, the truss is completely optimized in terms of shape. Note that this truss is related to one of the
Pareto points. The results of this problem is shown as a Pareto chart in Fig. 6in which the optimized shape of three points from Pareto points can be seen. The weight and deflection of these three points are presented in Table 1. It is seen that as the bars and height of the structure decreases, its weight decreases and the vertical deflection of the node 1 increases. Also, the more circular the structure is, its deflection decreases and its weight increases. The results of the shape optimization of Michel structure considering the weight and vertical deflection functions of the node 1 based on MOPSO method and shape evolutionary method are compared with each other in Table 2 In this table, the minimum vertical deflection of node 1 and the minimum weight of the structure based on MOPSO and evolutionary methods and also the primary weight and deflection of the structure are presented.

By this optimization, the truss' weight has a decrease of $23 \%$ from 78.6 to 60.43 kilogram and the deflection of node 1 shows a decrease of $76 \%$ and it reached from 4.805 to 1.13 millimeter.

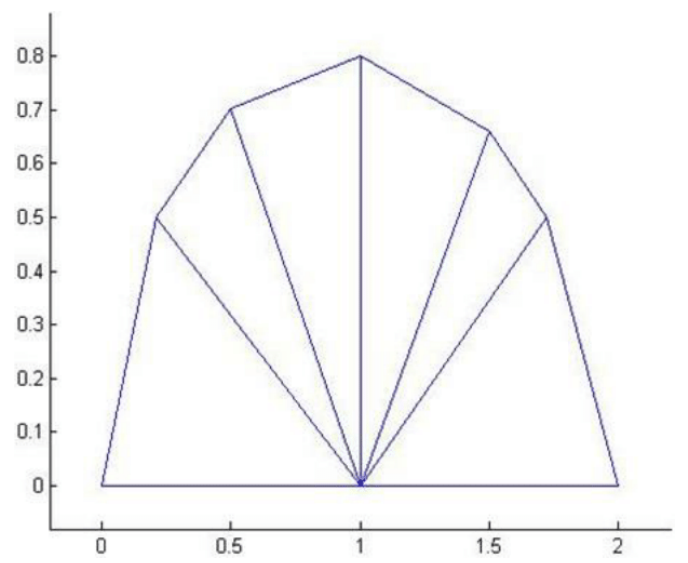

Fig. 5. Dimensions of one of the optimized trusses of the second problem according to meter

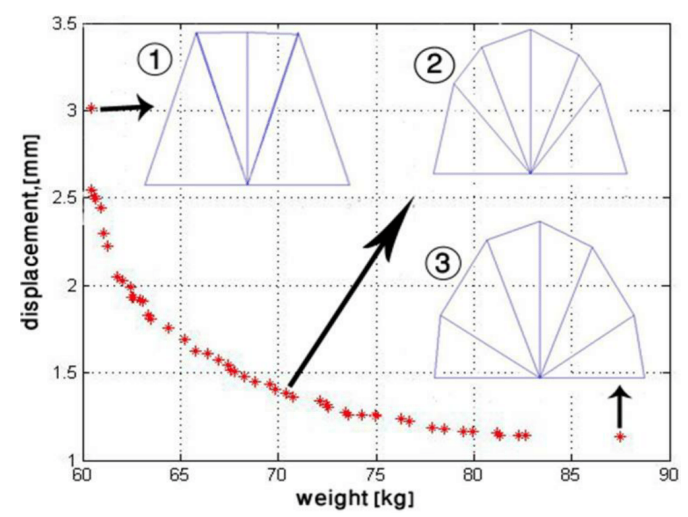

Fig. 6. Results and Pareto curve of the second problem

Tab. 1. Weight and vertical leap of node 1 for three points of the second problem's Pareto points (Michel structure)

\begin{tabular}{ccc}
\hline Truss & Weight $(\mathrm{kg})$ & Deflection $(\mathrm{mm})$ \\
\hline 1 & 60.43 & 3.02 \\
2 & 70.73 & 1.36 \\
3 & 87.47 & 1.13 \\
\hline
\end{tabular}


Tab. 2. Results of optimized structure based on MOPSO and evolutionary methods

\begin{tabular}{cccc}
\hline & Initial structure & MOPSO method & Evolution method 13 \\
\hline Minimum weight & 71.4 & 60.43 & 70.5 \\
Minimum deflection & 4.80 & 1.132 & 1.168 \\
\hline
\end{tabular}

Tab. 3. Complete characteristics of 18-bartruss of the third problem [15]

\begin{tabular}{|c|c|c|}
\hline Design variables & Shape & $x_{3} ; y_{3} ; x_{5} ; y_{5} ; x_{7} ; y_{7} ; x_{9} ; y_{9}$ \\
\hline Design variables & Size & $\begin{array}{c}a_{1}=a_{4}=a_{8}=a_{12}=a_{16} \\
a_{2}=a_{6}=a_{10}=a_{14}=a_{18} \\
a_{3}=a_{7}=a_{11}=a_{15} \\
a_{5}=a_{9}=a_{13}=a_{17}\end{array}$ \\
\hline Constraints & $\begin{array}{c}\text { Stress } \\
\text { Displacement }\end{array}$ & $\begin{array}{c}\left|\sigma_{i}\right| \geq 137,9 \mathrm{MPa}(20 \mathrm{ksi}) ; i=1,2, \ldots, 18 \\
-\end{array}$ \\
\hline Search Range & Shape Variables & $\begin{array}{c}-571.5 \mathrm{~cm} \geq y_{3}, y_{5}, y_{7}, y_{9} \geq 622.3 \mathrm{~cm} \\
1968.5 \mathrm{~cm} \geq x_{3} \geq 3111.5 \mathrm{~cm} \\
1333.5 \mathrm{~cm} \geq x_{5} \geq 2476.5 \mathrm{~cm} \\
698.5 \mathrm{~cm} \geq x_{7}<1847.5 \mathrm{~cm} \\
63.5 \mathrm{~cm} \geq x_{9} \geq 1206.5 \mathrm{~cm}\end{array}$ \\
\hline Search Range & Size Variables & $\begin{array}{c}8.89 \mathrm{~cm}(3.5 \mathrm{in}) \geq a_{i} \geq 50.8 \mathrm{~cm}(20 \mathrm{in}): \\
i=1,2, \ldots, 18\end{array}$ \\
\hline Loading & $\begin{array}{c}\text { Nodes: } \\
1,2,4,6,8\end{array}$ & $\begin{array}{c}F_{x}=0 \mathrm{KN}(\mathrm{kips}) \\
F_{y}=-89,075 \mathrm{KN}(-20 \mathrm{kips}) \\
F_{z}=0 \mathrm{KN}(\mathrm{kips})\end{array}$ \\
\hline Mechanical Properties & Density of the material & $\rho=0.0272 \mathrm{~N} / \mathrm{cm}^{3} \quad\left(0,1 \mathrm{lb} / \mathrm{in}^{3}\right)$ \\
\hline Mechanical Properties & Modulus of elasticity & $E=68.95 \mathrm{GPa}\left(1 \times 10^{4} \mathrm{q}, \mathrm{ksi}\right)$ \\
\hline
\end{tabular}

In this table $x_{i}, y_{i}$ are location of truss nodes and $a_{i}$ is cross section of each elements.

\subsection{Optimization of shape and size of an 18-bar truss [15]}

The next example is the truss shown in Fig. 7 In this figure $a=635 \mathrm{~cm}$ and the complete characteristics of this structure is presented in Table 3. The problem objective functions are weight optimization and maximum deflection of joints of this 18-bar truss, by optimizing the cross section and bars length simultaneously. After optimizing this structure, the results of simultaneous optimization of size and shape are presented and compared in Fig. 8. As it can be seen in Fig. 8, in some cases FSD-ES method can compete with MOPSO method in terms of weight, but is far behind in term of deflection. In other words, as can be seen in Fig. 8 the result from optimizing the shape and size of 2-dimensional truss is a one-objective answer by FSDES method along the Pareto curve related to multi objective optimization of size and shape of 2-dimensional truss By MOPSO method It confirms the accuracy of the presented Pareto curve. Since we could not find any similar examples to verify our results, we have compared single point of Pareto curve with single objective optimization done by others.

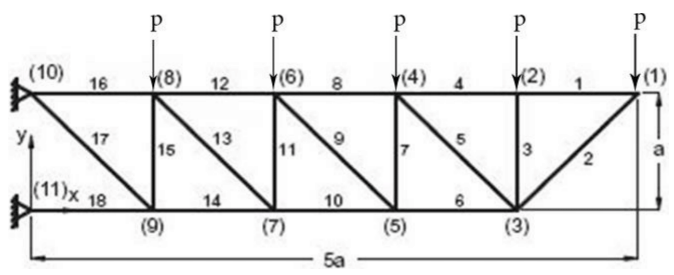

Fig. 7. 18-bar truss

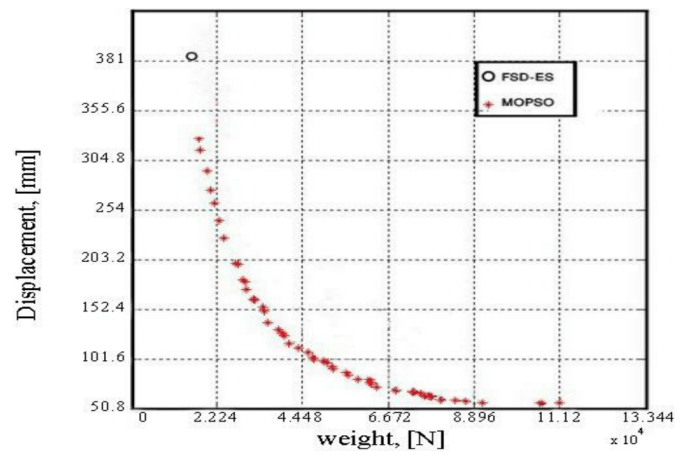

Fig. 8. Results of 18-bar truss

\section{Conclusions}

There are several methods to design and optimization of the truss structures. Some of these methods optimize the shape and size of the trusses with only one objective function. To the best of knowledge of authors the simultaneous optimization of shape and size of 2-dimensional truss through multi objective MOPSO method has not been done before. In this paper a multi objective method is presented for optimization. To test the accuracy and efficiency of method several examples are tested. As it is shown from the results, the answers achieved by MOPSO method are more accurate. The comparison of the results of this method with other methods, presented in literature, shows the better accuracy and desirable verity of pareto front. 


\section{References}

1 Schmit L, A Structural synthesis - its Genesis and Developments, IALAA journal, 19(0), (1981), 1249-1263, DOI 10.2514/3.7859

2 Rashidi A, Jazebi F, Brilakis I, Neuro-Fuzzy Genetic System for Selection of Construction Project Managers, ASCE Journal of Construction Engineering and Management, 137, (2011), 17-29, DOI 10.1061/(ASCE)CO.19437862.0000200

3 Kim Y, Park G, Nonlinear dynamic response structural optimization using equivalent static loads, J Comput Methods ApplMechEng, 199, (2010), 66076, DOI 10.1016 / j.cma.2009.10.014

4 Woo D, Choi J, Ali M, Jung H, A novel multimodal optimization algorithm applied to electromagnetic optimization, IEEE Trans Magn, 47, (2011), 1667-73, DOI 10.1109/TMAG.2011.2106218

5 Holland J, Adaptation in natural and artificial systems, Ann Arbor, MI: The University of Michigan Press, (1975).

6 Kaveh A, Talatahari S, A novel heuristic optimization method: charged system search, ActaMech, 213, (2010), 267-89, DOI 10.1007/S00707-0090270-4

7 Eberhart R, Kennedy J, A novel heuristic optimization method: charged system search, Proceedings of the sixth international symposium on micro machine and human science, In:, 1995, DOI 10.1109/MHS.1995.494215

8 Sheikhi M, Ghoddosian A, A hybrid imperialist competitive ant colony algorithm for optimum geometry design of frame structure, Structural engineering and mechanics, An int'l journal, 46(16), (2013), DOI 10.12989/sem.2013.46.3.403

9 Kennedy J, Eberhart R, Particle swarm optimization, proceedings of the IEEE international Conference on Neural Network, In:, 1995, pp. 19421945, DOI 10.1109/ICNN.1995.488968

10 Coello CA, Pulido GT, M.S. L, Handling multiple objectives with particle swarm optimization, IEEE Transactions on Evolutionary Computation, 8(3), (2004), 256-279, DOI 10.1109/TEVC.2004.826067

11 Guan-C L, Chung-H C, Multi objective optimal design of truss structure with immune algorithm, Computers and Structures, 82, (2004), 829-844, DOI 10.1016/j.compstruc.2004.03.003

12 Lamberti L, Pappalettere C, mproved sequential linear programming formulation for structural weight minimization, Comput Methods ApplMechEng, 193, (2004), 3493-521, DOI 10.1016/j.cma.2003.12.040.

13 Wang D, Zhang W, Jiang J, Truss shape optimization with multiple displacement constraints, Comput Methods ApplMechEng, 191, (2002), 3597 3612, DOI 10.1016/S0045-7825(02)00297-9

14 Wang D, Optimal shape design of a frame structure for minimization of maximum bending moment, EnginStruct, 29, (2007), 1824-1832, DOI 10.1016/j.engstruct.2006.10.004

15 Ahrari A, Atari A, Fully Stressed Design Evolution Strategy for Shape and shape optimization of Truss Structures, Computers and Structures, 123, (2013), 58-67, DOI 10.1016/j.compstruc.2013.04.013

16 Carlos A, Coello C, Salazar Lechuga M, MOPSO:A Proposal for MultipleObjective Swarm Optimization, Congress on Evolutionary Computation (CEC'2002), In:, Vol. 2, 2002, pp. 1051-1056, DOI 10.1109/CEC.2002.1004388

17 Kalyanmoy D, A FAST and Elitist Multiobjective Genetic Algorithm: NSGAII, IEEE TRANSACTIONS ON EVOLUTIONARY COMPUTATION, In:, Vol. 6, 2002, pp. 3597-3612, DOI 10.1109/4235.996017 